\title{
BMJ Open What does integrated care mean from an older person's perspective? A scoping review
}

\author{
Michael T. Lawless (D) , ${ }^{1}$ Amy Marshall, ${ }^{2}$ Manasi Murthy Mittinty (D) , ${ }^{3}$ \\ Gillian Harvey ${ }^{2}$
}

To cite: Lawless MT, Marshall A, Mittinty MM, et al. What does integrated care mean from an older person's perspective? A scoping review. BMJ Open 2020;10:e035157. doi:10.1136/ bmjopen-2019-035157

- Prepublication history for this paper is available online To view these files, please visit the journal online (http://dx.doi. org/10.1136/bmjopen-2019035157).

Received 21 October 2019 Revised 16 December 2019 Accepted 17 December 2019

Check for updates

(C) Author(s) (or their employer(s)) 2020. Re-use permitted under CC BY-NC. No commercial re-use. See rights and permissions. Published by BMJ.

${ }^{1}$ College of Nursing and Health Sciences, Flinders University, Bedford Park, South Australia, Australia

${ }^{2}$ Adelaide Nursing School, The University of Adelaide, Adelaide, South Australia, Australia ${ }^{3}$ Pain Management Research Institute, University of Sydney, Sydney, New South Wales, Australia

Correspondence to Dr Michael T. Lawless; michael.lawless@flinders. edu.au

\section{ABSTRACT}

Objective To systematically map and synthesise the literature on older adults' perceptions and experiences of integrated care.

Setting Various healthcare settings, including primary care, hospitals, allied health practices and emergency departments.

Participants Adults aged $\geq 60$ years.

Interventions Integrated (or similarly coordinated) healthcare.

Primary and secondary outcome measures Using scoping review methodology, four electronic databases (EMBASE, CINAHL, PubMed and ProQuest Dissertation and Theses) and the grey literature (Open Grey and Google Scholar) were searched to identify studies reporting on older adults' experiences of integrated care. Studies reporting on empirical, interpretive and critical research using any type of methodology were included. Four independent reviewers performed study selection, data extraction and analysis.

Results The initial search retrieved 436 articles, of which 30 were included in this review. Patients expressed a desire for continuity, both in terms of care relationships and management, seamless transitions between care services and/or settings, and coordinated care that delivers quick access, effective treatment, self-care support, respect for patient preferences, and involves carers and families

Conclusions Participants across the studies desired accessible, efficient and coordinated care that caters to their needs and preferences, while keeping in mind their rights and safety. This review highlights the salience of the relational, informational and organisational aspects of care from an older person's perspective. Findings are transferable and could be applied in various healthcare settings to derive patient-centred success measures that reflect the aspects of integrated care that are deemed important to older adults and their supporters.

\section{INTRODUCTION}

Evidence suggests that many older people are 'falling through the gaps' and experiencing fragmented care, ${ }^{1}$ particularly when they live with multimorbidity and frailty. In Australia, over $83 \%$ of the population aged over 75 years live with two or more chronic conditions $^{2}$ and, in the USA, around half of the

\section{Strengths and limitations of this study}

This is the first scoping review to identify, synthesise and appraise the quality of the available literature on older patients' experiences and perceptions of integrated care based on their journeys through the health system.

- Our review is comprehensive in nature, incorporating published peer-reviewed studies and grey literature on patient experiences of care integration.

- We used a narrative descriptive technique to synthesise the findings of the studies and extracted enablers and barriers to integrated care from an older person's perspective at the clinical, service and healthcare system levels.

- This review forms part of a larger body of research that aims to coproduce and evaluate locally relevant approaches designed to improve integrated care for older adults in South Australia.

- As this review incorporates studies drawing on different terms and definitions and reporting on different health conditions across a variety of healthcare settings, it may lack specificity.

population aged over 75 years is reported to live with three or more chronic conditions. ${ }^{3}$ This group commonly deals with health and functional challenges and reports almost twice as many problems resulting from poorly integrated care compared with those without multimorbidity. ${ }^{4}$ This is because they typically see several healthcare providers for different medical conditions, take multiple medications, have numerous agencies involved in providing care and experience a higher incidence of hospitalisation. ${ }^{5}$ These circumstances can compromise patient care, further contributing to poorer health outcomes, reduced quality of life and increased healthcare utilisation and costs.

Care integration is proposed as a solution to such fragmentation, ${ }^{6}$ with the potential to improve patient experiences while minimising unnecessary use of healthcare resources. Definitions and terminology used 
to describe integrated care differ within the published literature. The WHO defines integrated care, or integrated health services delivery, as:

An approach to strengthen people-centred health systems through the promotion of the comprehensive delivery of quality services across the life-course, designed according to the multidimensional needs of the population and the individual and delivered by a coordinated multidisciplinary team of providers working across settings and levels of care. ${ }^{7}$ (p 10)

Attempts to improve care integration have been made through numerous policy and research endeavours, yet the extent to which such efforts have achieved wide-scale impact remains questionable. A key message from research to date is that there is no 'one-size-fits-all' approach to integrated care. ${ }^{8}$ Rather, successful design and implementation of integrated care models requires attending to contextual factors, including local enablers and barriers. ${ }^{6}$ Evidence to date indicates that the most successful implementation efforts are: (1) bottom-up rather than top-down; (2) driven by local need; and (3) have the support and engagement of all key stakeholders, particularly patients and their carers/families. ${ }^{3}$ This suggests the need to engage older adults, their families and carers, and care providers to achieve the most effective care coordination and integration.

To date, relatively few studies have focused on patients' perspectives on integrated care, although some research suggests that there may be distinct differences between provider and patient narratives. ${ }^{9}$ Patients are more likely to emphasise the importance of relational aspects of care and the everyday consequences of living with their condition, as opposed to a clinical focus on managing specific health conditions. This reinforces the importance of understanding patients' perspectives and views of integrated care, rather than focusing primarily on policy and service-level priorities-a point highlighted in previous research ${ }^{10}$ and the focus of this scoping review.

To our knowledge, no evidence synthesis has summarised the available literature on older adults' views and expectations regarding integrated or similarly coordinated care. Starting with a focus on patient experiences rather than single-organisation or single-sector solutions, ${ }^{8}$ this review forms part of a larger programme of research that aims to coproduce and implement locally relevant approaches to improve integrated care for older adults at risk of repeated hospitalisation guided by a personcentred approach. ${ }^{1112}$

\section{Review questions}

The review protocol has been published previously ${ }^{12}$ and sets out a plan to address the following questions:

1. How do older patients define their views and experiences of integrated care?

2. What are the barriers and enablers of quality integrated care from an older person's perspective?
3. What is the quality of the literature on older patients' perspectives on integrated care?

4. What are the potential implications for the design and implementation of integrated care programmes for older people?

\section{MATERIALS AND METHODS}

Scoping reviews are used to understand the existing breadth of research on a topic, identify gaps in existing literature and assess the need for further investigation. ${ }^{1314}$ The scoping review methodology outlined by Arksey and $\mathrm{O}^{\prime}$ Malley ${ }^{13}$ was employed, details of which are published in our protocol. ${ }^{12}$ The Preferred Reporting Items for Systematic Reviews and Meta-Analyses extension for scoping reviews checklist ${ }^{15}$ was used to guide reporting.

\section{Identifying relevant studies}

The search strategy aimed to locate published peerreviewed studies and grey literature reporting on the views of older adults aged $\geq 60$ years (male or female) who had received integrated or similarly coordinated care of any definition in any type of healthcare setting. MMM performed the initial search in four electronic databases (EMBASE, CINAHL, PubMed and ProQuest) and two grey literature databases (Open Grey and Google Scholar). Studies published from June 2008 to July 2019 in English language were included to ensure feasibility and relevance to the current healthcare context, that is, studies conducted after the publication of a consensus definition of integrated care by the WHO.$^{12}$ No limitations were placed on study design, type of healthcare setting, geographical location, or the upper age and gender of the participants. Literature search strategies were developed using keywords pertinent to older patients and their perspectives. Appropriate variations in spelling and plurals were used in the search (table 1).

\section{Study selection}

Studies were selected via a three-step process. First, AM, GH and MMM independently screened titles and abstracts to determine inclusion status. A second screen of full-text articles (AM, ML) ensured that the studies met the inclusion criteria. Third, AM and ML assessed the remaining full-text records for eligibility. A third author (GH) assessed the articles when the other reviewers were uncertain about eligibility status. Disagreements between the reviewers were resolved through group discussion. Finally, we conducted bibliographic searching of the reference lists of the included articles to identify additional potentially relevant studies.

\section{Data extraction}

AM, ML and GH used a standardised form to extract relevant data and consulted regularly to help maintain uniformity during the extraction procedure. The following data were extracted: bibliographic information, aim(s) of study, additional research questions/ objectives, study design characteristics, participant 
Table 1 Search terms used for PubMed database

\begin{tabular}{|c|c|c|}
\hline Word group 1: older patients & Word group 2: patient perspectives & Word group 3: integrated care \\
\hline $\begin{array}{l}\text { 'Aged'[mh] } \\
\text { OR } \\
\text { older patient }{ }^{\star}[\mathrm{tw}] \\
\text { OR } \\
\text { elderly patient*[tw] }\end{array}$ & $\begin{array}{l}\text { views*[tw] } \\
\text { OR } \\
\text { perspective*[tw] } \\
\text { OR } \\
\text { expectation*[tw] } \\
\text { OR } \\
\text { experience } *[t w]\end{array}$ & 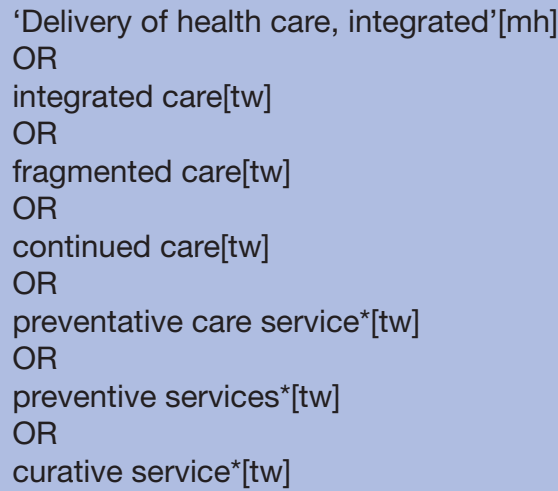 \\
\hline
\end{tabular}

characteristics, definition of integrated care provided, outcomes reported, most important findings containing patient voice, other relevant findings, conclusions, study limitations and author recommendations.

\section{Quality appraisal}

Although it is not customary to undertake a quality appraisal as part of a scoping review, ${ }^{13}$ because the purpose of this review was to inform recommendations for healthcare policy and practice, a formal quality assessment was deemed appropriate. The Joanna Briggs Institute critical appraisal checklists ${ }^{1617}$ were used to assess the quality of final studies included in the review. We used these checklists due to their brevity, clarity and explicit focus on appraising the voices of participants and their role in generating and/or interpreting the research findings. These tools are designed to assess the methodological quality of studies and determine the extent to which studies have addressed the possibility of bias in study design, conduct or analysis. ML, AM and GH independently assessed the papers; scores were categorised into 'low' (1-3/10 for qualitative research; 1-2/8 for quantitative research), 'average' (4-7/10; 3-5/8) and 'high' (8-10/10; 6-8/8) ranges for each assessment tool. These ranges were used to define an overall quality rating for each article. In line with scoping review methodology and in contrast to systematic review methodology, articles were not excluded on the basis of methodological quality assessment or intervention effectiveness.

\section{Reporting the results}

A narrative descriptive technique was used to synthesise the findings of the studies. ${ }^{18} 19$ Using this approach, we familiarised ourselves with the completed data extraction forms and inductively generated codes as they related to the review questions and aims. Studies and their findings were then grouped into logical categories and common themes were identified. Potential enablers and barriers were extracted from the synthesis and categorised into the corresponding thematic categories using a combined inductive and deductive approach. The final themes were decided on through deliberation and reference to supportive data. Analytical rigour was upheld through regular team meetings in which codes, categories and major themes were discussed and crosschecked with an audit trail maintained in a Microsoft Excel workbook.

\section{Patient and public involvement}

A local advisory group working with older adults was engaged periodically during the review process. ${ }^{12}$ To ensure a patient-centred approach and to facilitate the application of the review findings, results will be disseminated among patients, carers and other stakeholders and presented at public forums. Findings from this review will feed into ongoing research with the objective of coproducing and evaluating local initiatives designed to improve integrated care for older adults in South Australia.

\section{RESULTS}

\section{Search results}

Four hundred and thirty-six articles were retrieved from the initial search and, following the removal of duplicates $(n=100), 336$ articles were screened for eligibility. Fifty-six articles were selected for full-text review and, of these, 26 articles were included in the review. Reference list searching identified an additional four articles, which are included in the final review (figure 1).

\section{Description of studies}

Fifteen studies $(50 \%)$ used a qualitative methodology, ${ }^{20-34}$ while the remainder used a quantitative methodology $\left(20 \%, n=6^{35-40}\right)$, or a mixed methodology $\left(20 \%, n=6^{41-46}\right)$. Three records $(10 \%)$ were reports produced by two nongovernment organisations in the UK. ${ }^{47-49}$ Most of the studies were conducted in Europe $(53 \%, \mathrm{n}=16)$ or North America $(40 \%, \mathrm{n}=12)$. Sample sizes of patient groups or subgroups (ie, older adults) ranged from 4 to 15617 participants. The average age of participants in the older adult/patient groups ranged from 59 to 87 years. Although we focused on the experiences of older people, studies reporting on younger participants (ie, aged $<60$ years) were included if the average age of participants was $\geq 60$ years due to the limited number of eligible studies 

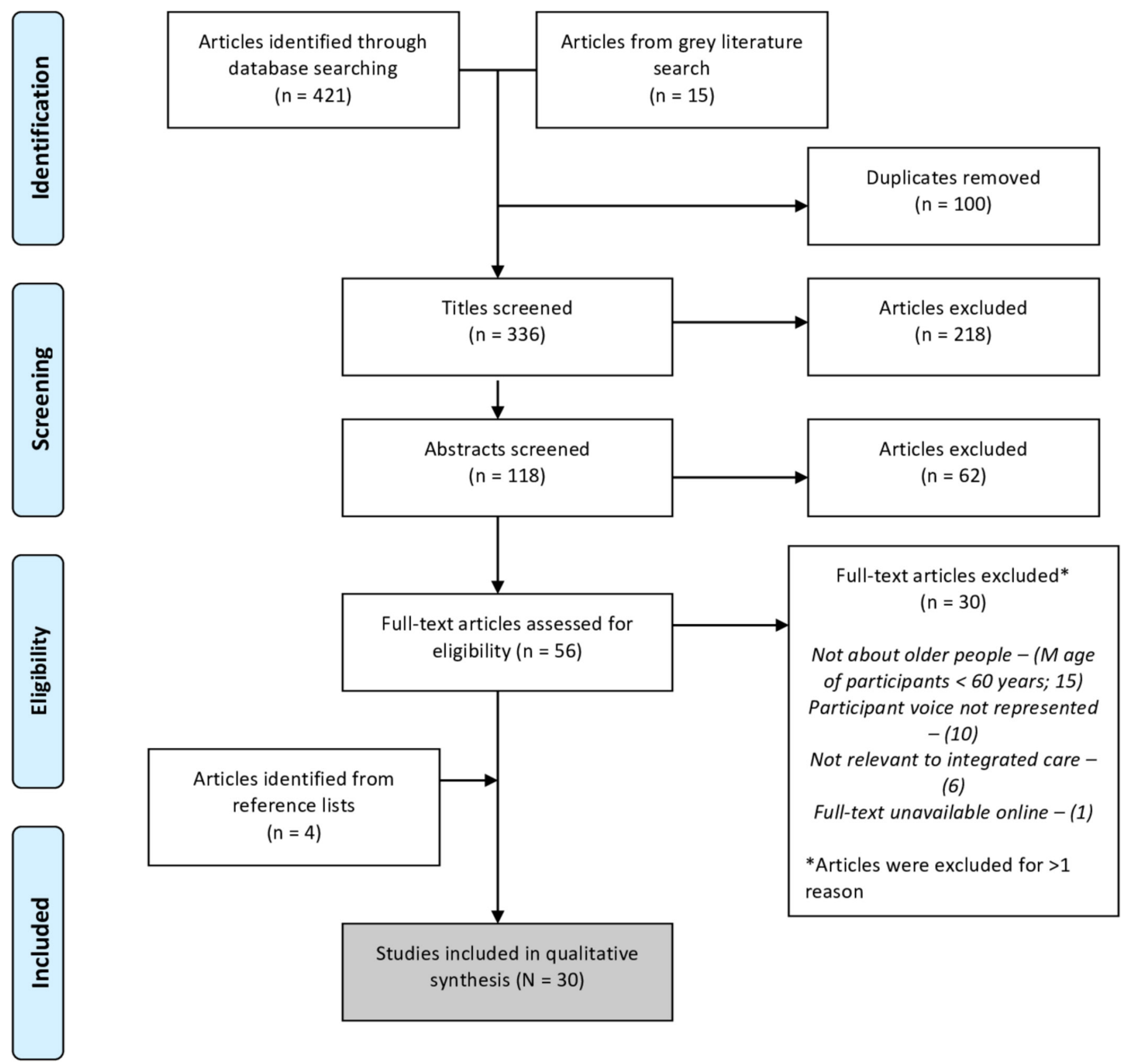

Figure 1 Preferred Reporting Items for Systematic Reviews and Meta-Analyse (PRISMA) flow diagram.

reporting exclusively on older people. Participants had one or more of the following conditions: chronic obstructive pulmonary disease, cancer, coronary heart disease, diabetes, stroke, arthritis and asthma (table 2).

Six articles $(20 \%)$ provided a definition of integrated care. $^{2026} 28304446$ Although definitions varied, they cited common elements and/or principles including: comprehensive services, coordinated care, patient focus, multidisciplinary and/or interprofessional teamwork, effective information systems, optimised resource use, and appropriate organisational culture and leadership. None of the studies explicitly referenced the WHO definition of integrated care. ${ }^{7}$ Reflecting the diffuse research literature on integrated care across academic journals and disciplines and the inconsistent terminology used to describe integrated care programmes, initiatives, settings and/or evaluations, ${ }^{50}$ several other concepts and terms were mentioned or defined in the articles in relation to care integration: "care coordination', 22273940 'continuity of care', 25323847 'shared care', 'collaborative self-management', ${ }^{43}$ 'person-/patientcentred care,. 242936373941

\section{Quality of the included articles}

The methodological quality of the included studies was rated as 'average' (15 studies) to 'high' (12 studies); quality appraisal was not performed on the three reports. Common limitations of the qualitative papers were: lack 


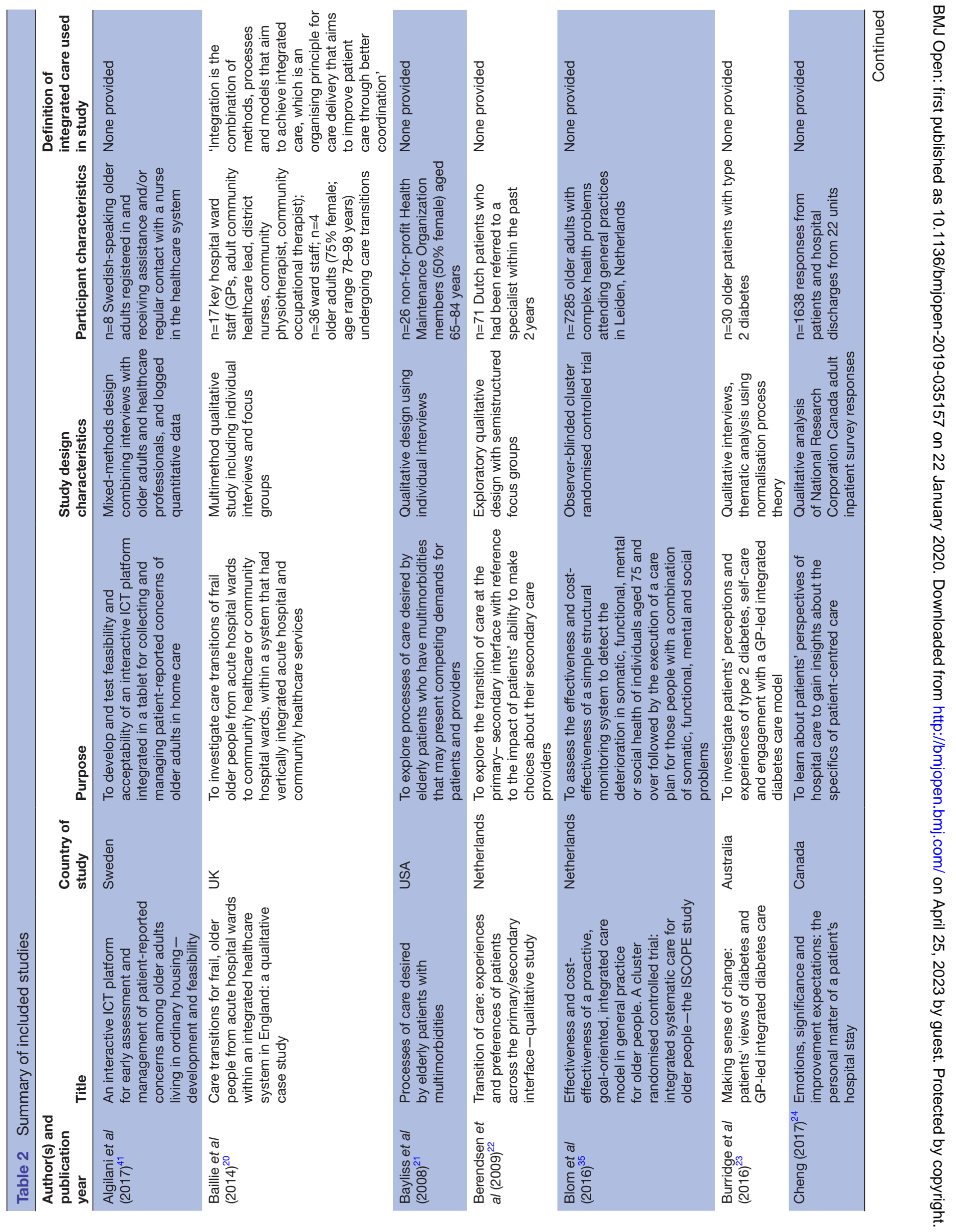




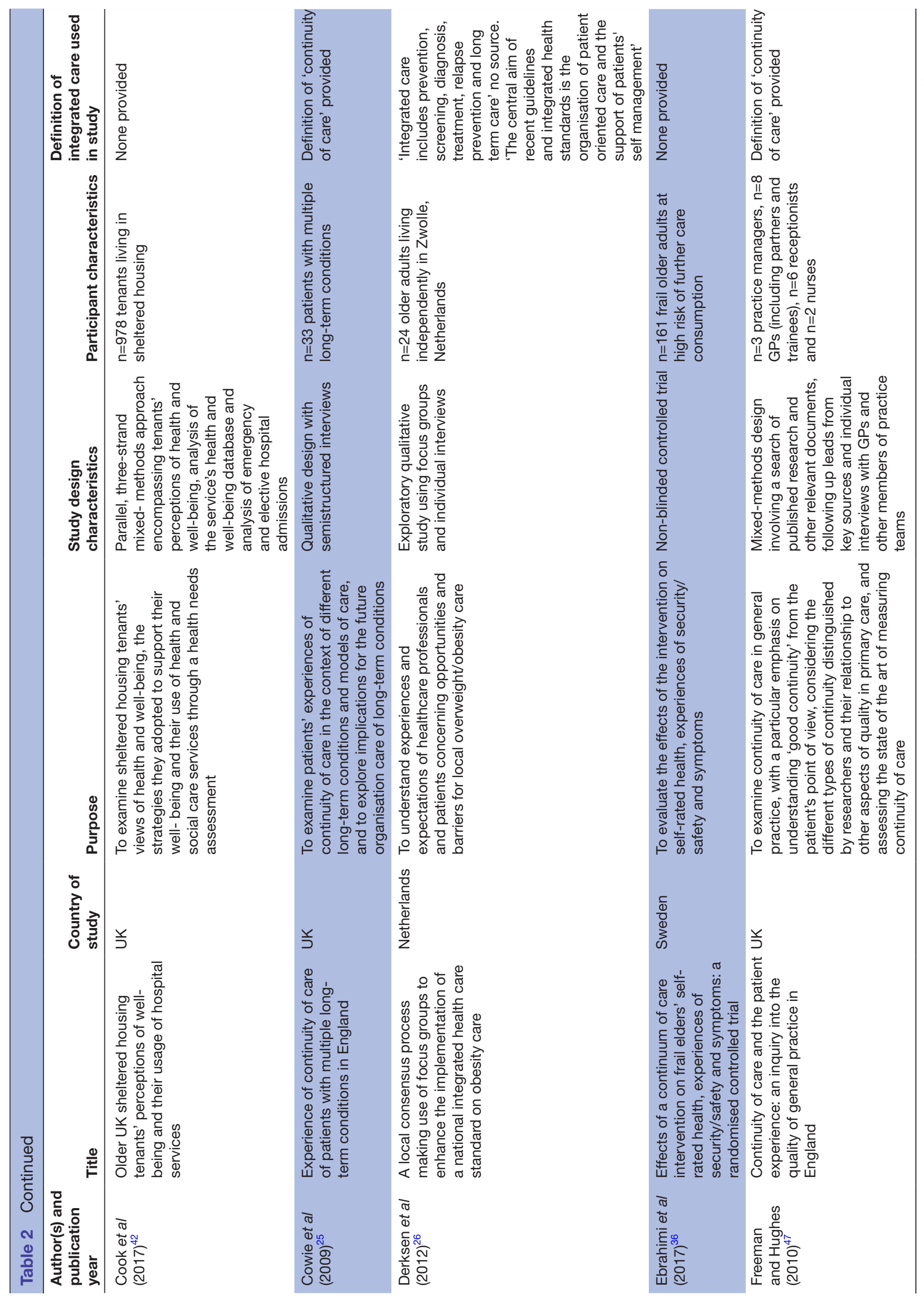




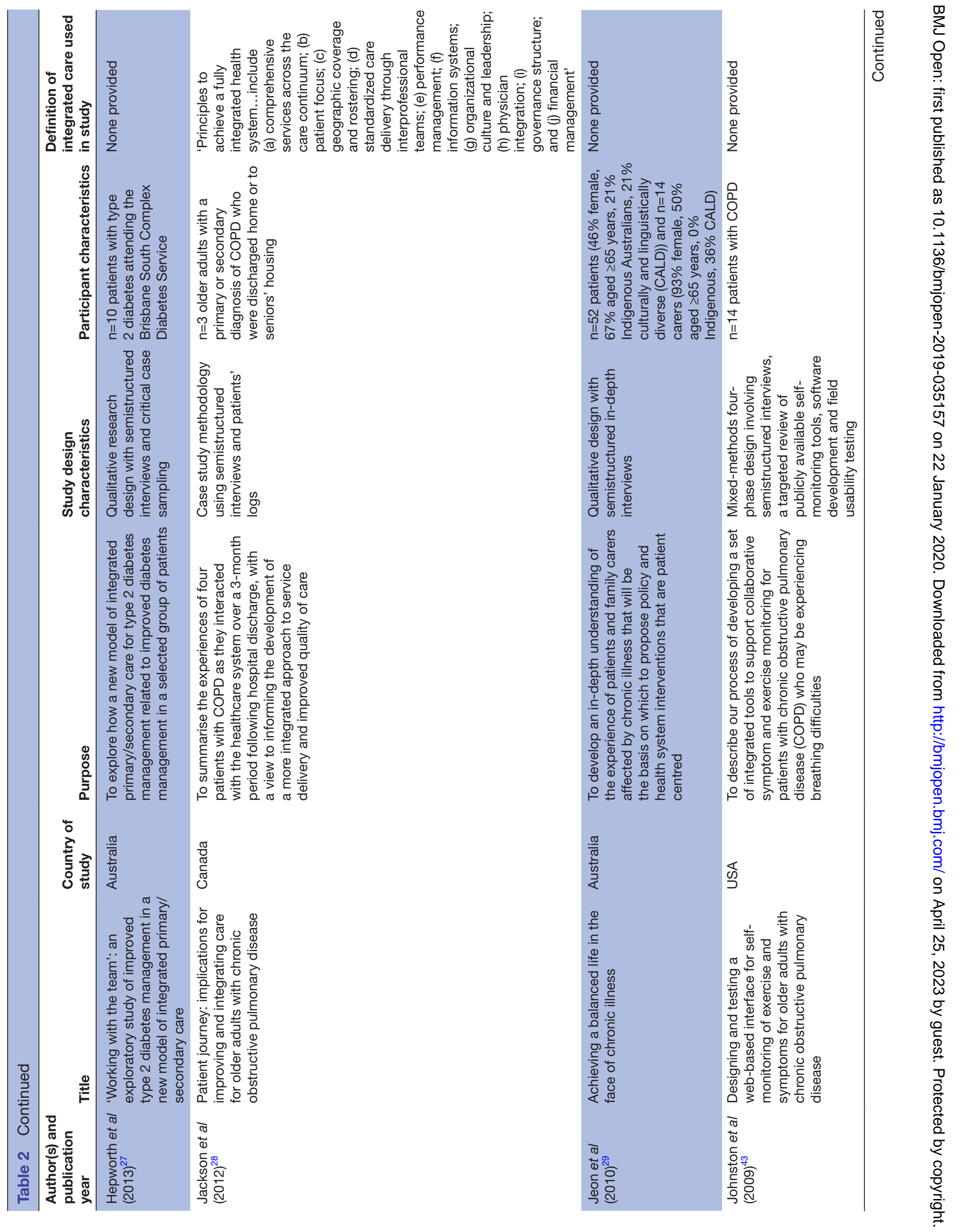




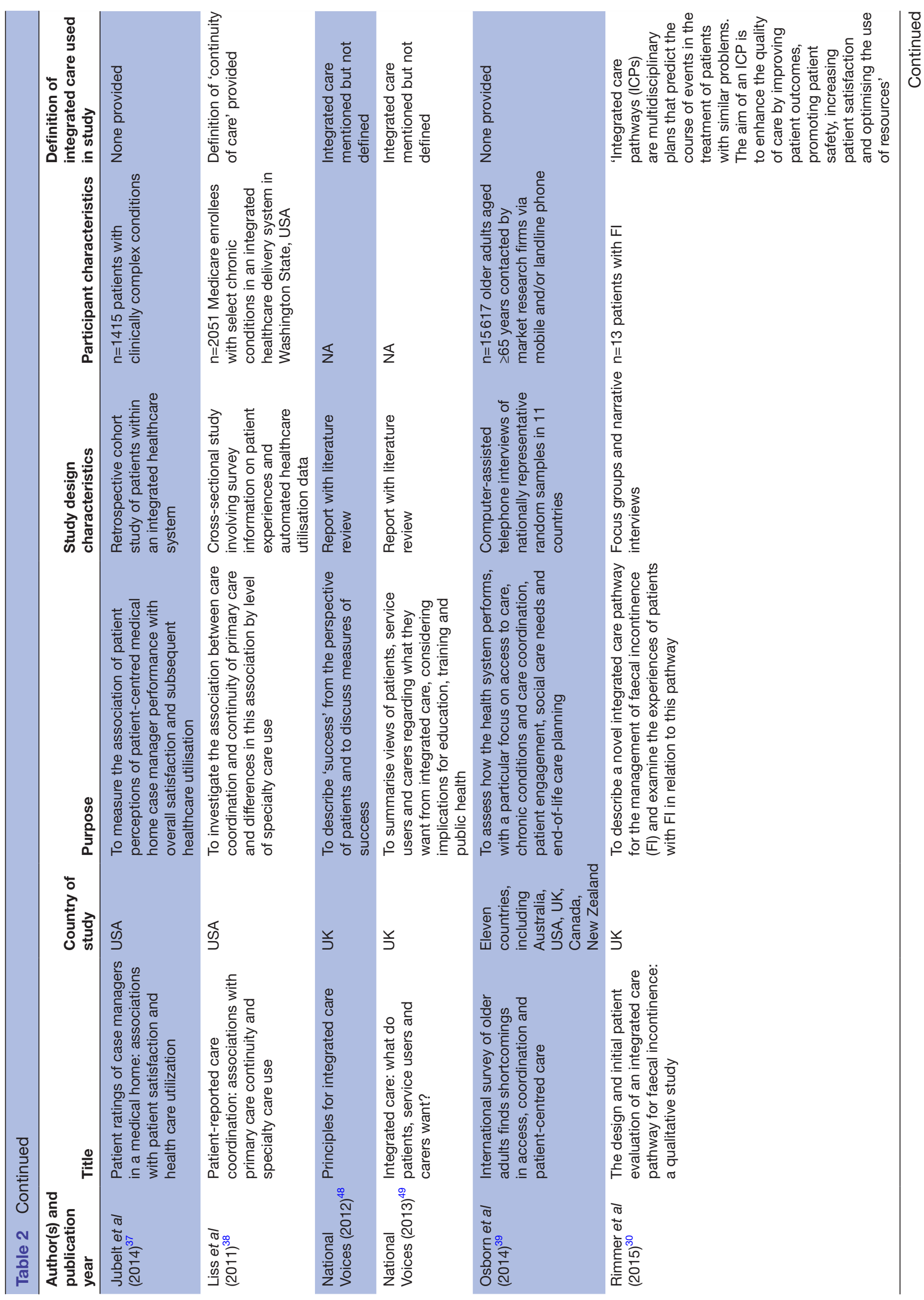




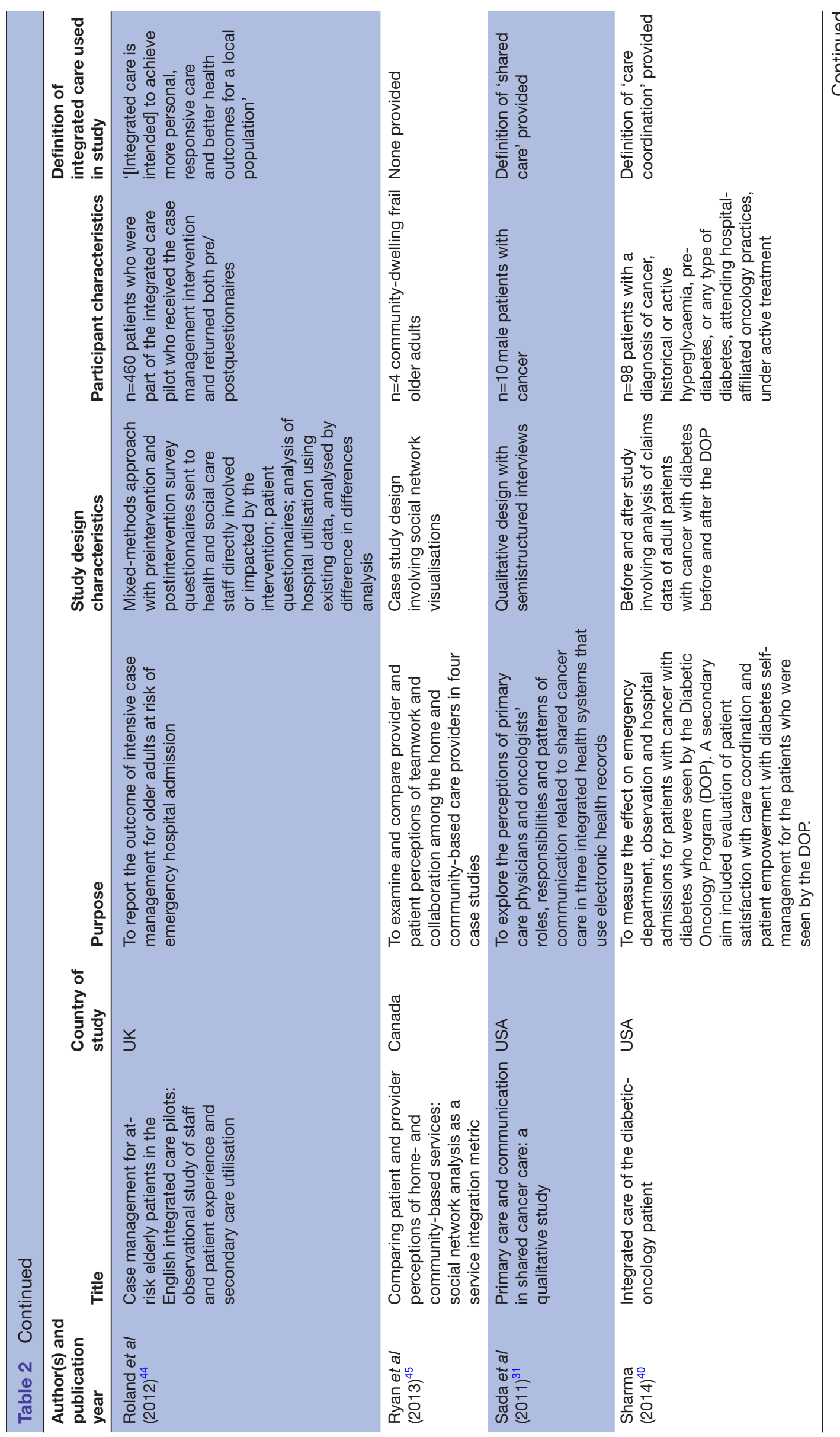




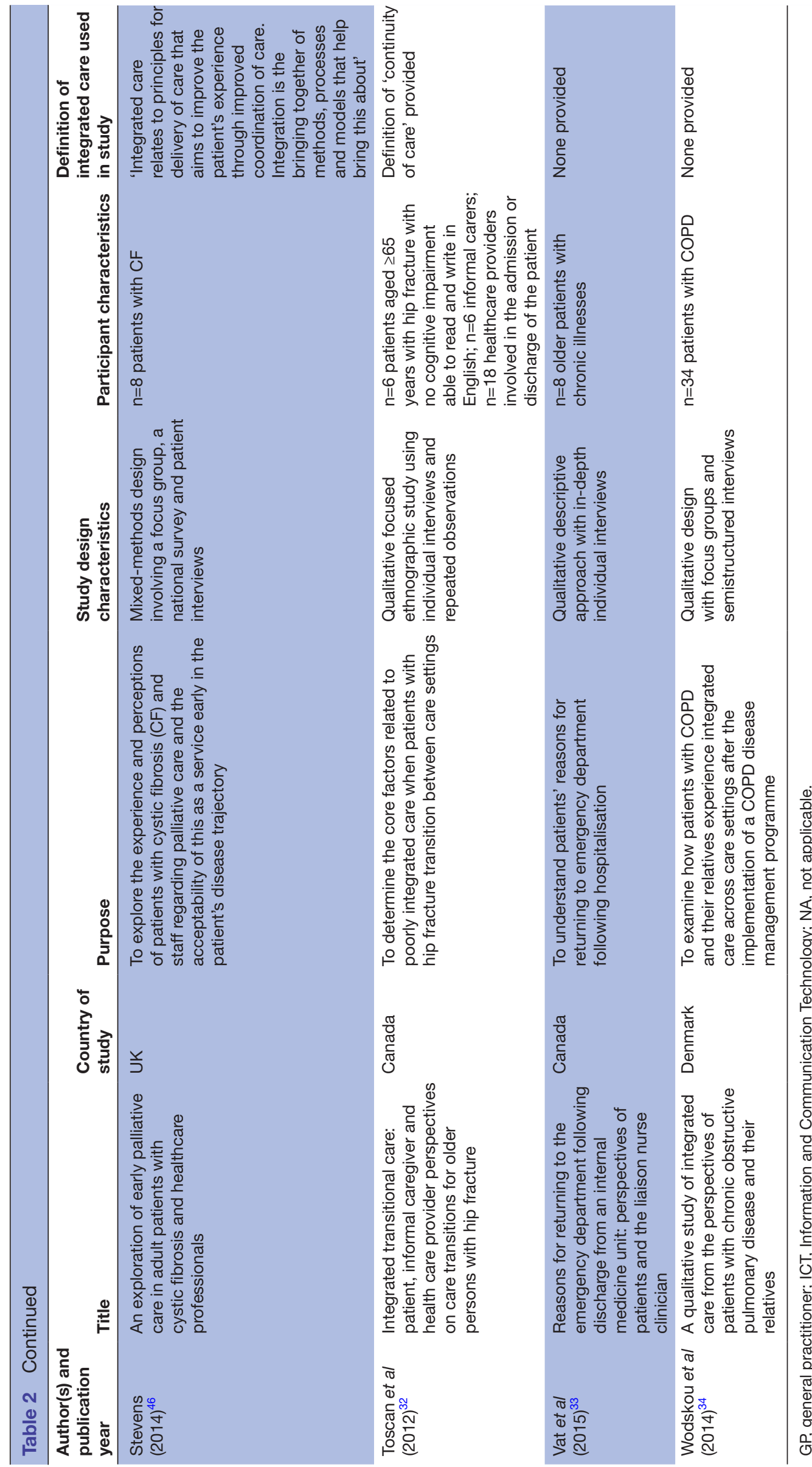


of acknowledgement of the influence of the researcher on the research or vice versa ${ }^{20-23}$ 25-28 303133343740414345 ; lack of a statement about the cultural or theoretical position of the researcherr-23 25-27 29-31 333437414345 ; and a lack of congruity between the stated philosophical or theoretical perspective and the research methodology. ${ }^{21} 22$ 24-27 29-34 3740 For quantitative studies, the most common limitation was inadequate consideration of confounding factors $(100 \%)$.

\section{Synthesis of findings}

Five themes were identified reflecting older adults' experiences and views of integrated care: (1) access and availability; (2) involvement, initiative and follow-up; (3) communication and information; (4) referral and care transitions; and (5) coordination and cooperation.

\section{Access and availability}

Participants in 10 studies identified the ability to access healthcare providers and services as critical. ${ }^{21} 25$ 27-29 3436414749 Access was generally discussed in relation to: physical accessibility, ${ }^{21} 252834$ convenient access to a known and trusted professional when needed $^{212528344749}$; and access difficulties due to service eligibility restrictions, lack of formal home support and/ or the unavailability of needed services or supports in different geographical locations. ${ }^{28} 34{ }^{49}$ Three papers discussed difficulties associated with the physical accessibility of services. ${ }^{25}{ }^{28}$ Problems related to physical access caused significant anxiety for participants, especially when these issues were compounded by unexpectedly long waiting times. ${ }^{25}$

Access was discussed in relation to the continuity of relationships in 10 articles. $^{21} 2329$ 34-36 42-44 48 Participants generally spoke of relationship continuity in terms of the establishment and maintenance of relationships between patients, their carers/families and a known and trusted health or social care professional, as well as positive health and psychosocial impacts of such relationships. ${ }^{22} 232547$ One report ${ }^{47}$ stated that patients 'have clear preferences' regarding seeing a familiar healthcare professional, such as a general practitioner (GP), giving this preference greater priority still when problems are chronic or distressing. Although patients valued having a long-standing relationship with a single provider, they were prepared to forgo seeing a familiar provider in favour of quick access. ${ }^{47}$

Nine studies discussed the applicability of technologies as part of integrated care approaches for older populations. ${ }^{21} 2831343541434849$ Participants viewed technology as having an important role, particularly when they experienced difficulty in accessing services, when care plans became increasingly complex and/or were updated repeatedly, and when additional information was needed urgently. ${ }^{21} 4849$ Three studies described technical and experiential factors associated with the successful implementation of technologies. ${ }^{35} 4143$ Participants in these studies identified user-friendliness ${ }^{34}$ and supported self-care $^{41}{ }^{43}$ as key factors influencing their views on specific technologies.

\section{Involvement, initiative and follow-up}

Participants wanted to be involved in decisions about their care and treatment in accordance with their needs, preferences and capacities at the time of the encounter. ${ }^{2022} 26283234394849$ Although participants generally expected to be involved in decisions regarding their care, treatment and medicines, ${ }^{49}$ they often felt that care was not 'centred' on them. ${ }^{32}$ Some participants did not want, or were unable, to make their own healthcare decisions, stating that they preferred to leave decisions up to their family doctor, particularly those related to referral. ${ }^{22}$ Others expressed that although they preferred their family doctor to consult with them, they ultimately wanted to be kept informed and given the opportunity to make their own decisions with the support of health professionals. ${ }^{22} 2849$ Participants felt that there was less scope for them to make decisions when consulting with specialists ${ }^{26}$ or when planning for discharge from hospital. ${ }^{20}$

The importance of initiative and follow-up was discussed in seven articles. ${ }^{22} 262834444849$ Participants expected providers to demonstrate initiative by being knowledgeable about their condition and the patient 'as a person', ${ }^{49}$ considering the applicability of diagnostic investigation, regularly reviewing patients' care and treatment and opening up discussions about referral and/or patients' home care needs. ${ }^{348}$ Participants expected providers to take responsibility for following up on previously initiated actions. Indicators of lack of follow-up included missing test results, sudden termination of home care and support without a needs reassessment and serious health conditions remaining untreated for a significant period of time following initial diagnosis. ${ }^{34}$

Participants' views on the involvement and needs of carers and families varied. ${ }^{2022} 28324849$ Participants appreciated when carers accompanied them to appointments with healthcare professionals because it assisted them with comprehension, remembering care instructions, scheduling future appointments and providing personal health information when necessary. ${ }^{28}$ This was sometimes difficult, however, as carers were generally 'further removed' from the dissemination of information. ${ }^{32}$ As a result, confusion often existed between professionals and carers about individual roles and responsibilities, resulting in 'blurred boundaries' and ambiguity in information sharing and flow. Patients considered it important to attend to carers' informational and emotional needs, for example, by providing them with instructions about disease management and assessing their stress tolerance. ${ }^{22}$

\section{Communication and information}

Older adults expected highly developed communication skills and clear, comprehensive information from all providers with whom they interacted regardless of their condition(s), the care setting or the provider's qualification. ${ }^{21} 2224$ 26-28 3032344749 Clarity, attentiveness, empathy 
and respect were generally considered to be important elements of 'good' patient-provider communication and relationships. ${ }^{21} 22$ 24-28 344647 Participants expected providers to demonstrate these traits/competencies by: listening carefully to patients' perspectives and preferences; informing patients of the relative advantages and disadvantages of referrals and treatments; providing personalised care (ie, not treating patients like a number ${ }^{4649}$ ); using appropriate and accessible language; taking patients' concerns seriously; and responding empathetically to patients' emotions. These actions were reported to enable open communication and shared decision-making, ${ }^{34}$ increase patients' motivation to engage in healthy lifestyle practices ${ }^{26}$ and help patients feel supported and 'cared for'. ${ }^{24}{ }^{276}$ Failure to recognise and respond to patients' emotions was seen to undermine the therapeutic relationship and compromise patients' perceptions of safety and care quality. ${ }^{24} 34$

Information was seen as vitally important as it enabled and mediated older patients' interactions with the healthcare system. ${ }^{22} 2528344849$ Participants expressed satisfaction with the information they received from providers, particularly when information was provided at important junctures in their care journey, and was followed up promptly and consistently. ${ }^{34} 4049$ Having a care plan on record, and having knowledge of its contents and updates, was associated with more positive patient experiences, particularly in terms of feeling involved in healthcare decision-making and care processes. ${ }^{48}$ Patients disliked having to 'repeat their story' to multiple providers-this was cited as a main reason for wanting to see a familiar healthcare provider. ${ }^{47}$ Participants appreciated when providers informed them of other available services to which they might be entitled, as well as information about how to manage financially. ${ }^{49}$ Participants additionally desired information that could help them comprehend and prepare for the impacts of their health conditions on other aspects of their life.

Participants' preferences regarding sources of information were inconsistent. ${ }^{22} 34$ Some participants preferred contact with specialists due to their expertise and prior negative experiences of family doctors providing insufficient information to address their health concerns. A majority of participants, however, preferred to receive information from a primary care provider, such as a GP, in the first instance. Nurses were identified as a valued information source due to their perceived capacity to provide extensive and comprehensible information. ${ }^{34}$ Supplementary (usually written) information was appreciated, and was seen as particularly useful when the presence of multimorbidity increased the complexity of developing, understanding and executing care plans. ${ }^{21}$ Participants felt it was important to be able to view their health records at any time in order to determine who to share this information with and correct any misinformation. ${ }^{49}$

Problems related to information sharing and transfer were discussed in seven articles. ${ }^{20} 222532343949$ Participants felt that different providers across the primary-secondary care interface often had conflicting information about, and opinions of, their care. Missing or conflicting information caused a great deal of uncertainty and confusion for patients and their caregivers. Relatedly, incomplete transfer and availability of relevant information to other healthcare providers was identified as a common interorganisational and intraorganisational barrier that could lead to fragmented care, confusion or dissatisfaction. ${ }^{25}$ In hospital, some participants experienced conflicting information about discharge and were unaware which ward staff (if any) were planning their discharge,${ }^{20}$ sometimes due to the absence of a written discharge plan. ${ }^{39}$

\section{Referral and care transitions}

Transitions between services and care settings were generally seen as significant points at which older patients were particularly susceptible to lapses or losses of continuity. ${ }^{202} 2728303247-49$ Timely and appropriate referral was therefore perceived to be essential; participants appreciated when their family doctor was able to minimise the time between referral and their first consultation. ${ }^{22}{ }^{30}{ }^{48}$ Patients expected their primary care provider to be aware of their hospital treatment and be informed of the outcome of any investigations. ${ }^{47}$ The impacts of inappropriate referrals and/or poorly managed transitions were particularly apparent for patients with multimorbidity who typically received care from multiple professionals/services at different stages of the illness trajectory. Patients identified timely availability of information, effective planning and communication as key elements of well-coordinated care, as they provided a tangible sense of 'being handed over' from one setting/ service to another. ${ }^{47}$ A lack of clarity about who should be responsible for managing information was cited as a major reason for information being lost when patients and their personal information are transferred between settings. ${ }^{32}$ Patients in the same study felt that a sense of 'diluted ownership' of care delivery and outcomes caused them to disengage from the management of their own care, leading to losses of personal autonomy.

When participants were not referred to services promptly, they tended to presume that providers were unaware of those services. ${ }^{30}$ Inadequate promotion of services among providers and the public was identified as a possible reason for delayed referral or non-referral. ${ }^{30}$ When moving to a new service, participants desired to be: informed in advance where they were going and the name of their primary contact person; assured that information about their views and preferences, and any agreed care plan, was passed on in advance; and given the flexibility to continue to see, as appropriate, preferred healthcare professionals who knew them and their situation well. ${ }^{48} 49$ Participants stressed the need to preserve entitlements to care despite movements between services and across geographical boundaries.

Concern about waiting times during care transitions and referrals featured in six articles. ${ }^{20} 22$ 25 32-34 49 Participants felt that minimising the length of waiting time during periods of transition was important, particularly in 
the hospital setting or when waiting for diagnostic investigation, ${ }^{20} 2249$ but expressed concerned about 'being rushed', particularly during periods of recovery or when facing significant decisions about their care and/or treatment. ${ }^{32}$ Participants from two studies recounted negative experiences of poor care coordination between providers and services, with some explaining that they had waited up to a year before seeing a specialist following referral from their GP. ${ }^{25}{ }^{34}$ Lengthy wait periods caused feelings of uncertainty, frustration and abandonment, particularly if symptoms were perceived as worsening and/or beyond patients' self-management abilities. ${ }^{2122}$

\section{Coordination and cooperation}

Twelve studies discussed the importance of cooperation, coordination and communication across organisations, between service providers and across types of care. $^{20} 2225-2730343845464849$ Although participants generally had minimal interest in institutional/organisational priorities regarding integrated care, they wanted professionals and services to "work together as a team around the patient ${ }^{\prime} .{ }^{49}$ Divisions between primary, secondary and community care were regarded as relatively meaningless compared with patients' overarching desire for highquality care and continuity, regardless of the source and/ or setting. ${ }^{49}$

Participants were generally satisfied with their care when: they were referred to services without difficulty ${ }^{34}$; interprofessional communication was perceptible and shared with the patient, ${ }^{49}$ and discharge processes were perceived as consultative and coordinated. ${ }^{20}$ However, the fragmented nature of the healthcare system became apparent to patients when they needed to contact multiple providers to coordinate care, errors occurred between care transitions, or when providers disagreed on necessary care and services. ${ }^{34}$ Coordination and cooperation was also discussed in relation to resources, patient rights and entitlements regarding support and financing across organisations and care settings. ${ }^{49}$

In cases where little teamwork was perceived, participants tended to experience inappropriate, inefficient or inconsistent referrals, reduced motivation to comply with treatment and ineffective or inappropriate responses to emergent needs or unanticipated problems. ${ }^{25} 263445$ Participants viewed serious communication breakdowns (eg, failure to record drug allergies) as unacceptable and damaging to their health. ${ }^{25}$ Patients living with severe illness and/or those with multiple health problems were seen as suffering most from insufficient interprofessional cooperation. ${ }^{34}$

Four studies identified a possible tension between increased specialty care use and primary care providers' capacity to coordinate care. ${ }^{22} 283438$ Some participants recounted that although communication between their primary care provider and specialist was evident around the time of referral and the initial specialist appointment, they were unsure whether ongoing communication and follow-up was occurring. ${ }^{22} 28$ Participants perceived that visits to specialist care added further complexity to the care delivery process and presented opportunities for gaps in care coordination to occur. ${ }^{22}$

The appointment of a care coordinator, also referred to as a case manager, was proposed as a solution to poor access, follow-up and coordination between organisations, sites, or providers in six studies. ${ }^{34} 3637444749$ Functions of care coordinators described by participants included: acting as a primary contact person and 'main person' responsible for patients' care, particularly aged and frail patients with serious and chronic conditions; coordinating care and social services across different agencies within the healthcare system; regularly contacting patients and their families; offering guidance about symptom management, and providing assistance with instrumental activities of daily life. $^{344749}$

\section{DISCUSSION}

This review highlights that older adults typically define their perspectives towards integrated care with respect to the relational, informational and organisational aspects of care. These aspects of care and types of continuity were considered to be important drivers and benchmarks of person-centred integrated care, and were central features of patients' diverse narratives. Our findings concur with previous research exploring the semantic misalignments between patient and medical narratives and understandings of person-centred care coordination. ${ }^{951}$

\section{Enablers and barriers from the perspective of older patients}

Based on our synthesis of patient perspectives, several enablers and barriers were identified. Key enablers included: access arrangements that reflect patient needs and preferences regarding which services to access, the speed of access and the methods of access, ${ }^{21} 25$ 27-29 34364149 appropriate user-friendly technologies, ${ }^{2841}$ clear communication coupled with appropriate information, ${ }^{22} 24$ 26-28 3034 regular contact with a familiar, trusted healthcare provider, ${ }^{22-24}$ individualised care planning with appropriate patient involvement in healthcare decision-making ${ }^{28} 34$ and systems to reduce gaps in information and to enable regular follow-up. ${ }^{22} 283436$

Barriers to integrated care as defined by older adults included: unavailability of needed providers and services in certain areas/jurisdictions, ${ }^{28} 3449$ lack of opportunities to clarify patients' needs, priorities and preferences, ${ }^{22} 2734$ including those related to patients' information and/ or communication, ${ }^{22} 25283447-49$ conflicting information, clinical advice, treatments and/or management, ${ }^{22} 25344849$ lengthy wait times, ${ }^{2022} 2533344749$ limited interprofessional or multidisciplinary teamwork ${ }^{2526344547-49}$ and relational and informational discontinuity at the primary-secondary care interface. $2022283234393947-49$

Defining the meaning of integrated care from the perspectives of different stakeholders, and reconciling those perspectives, poses considerable policy and research challenges. ${ }^{52}$ Structural and organisational-based 
definitions are well founded insofar as they are driven by the need to overcome sectoral fragmentation in healthcare systems ${ }^{53}$ However, such concerns are likely to be less important to patients than their smooth, seamless and supported journey through the care system. ${ }^{48}{ }^{49}$ Organisationally based definitions can overshadow personcentred understandings of care integration, which are needed to guide the delivery of appropriate, coordinated and responsive care. Person-centred definitions based on patient experiences capture a fundamental principal of integrated care, and offer a cogent logic as to its key objectives and success measures.

\section{Implications for implementation and knowledge translation}

Our findings suggest a need to better prepare and support providers across the healthcare system to deliver coordinated, efficient and appropriate person-centred care within a complex milieu. The successful implementation of integrated care into practice requires providers to internalise the value of the principles of integrated care while being open to new ways of working within non-traditional models of care delivery. ${ }^{154}$ Implementing sustainable integrated care systems for older adults will require multipronged, transformative action at the clinical (eg, facilitating shared decision-making and goal setting) service (eg, supporting the coordination of services delivered by multidisciplinary providers) and healthcare system (eg, strengthening governance, accountability systems and financing mechanisms enabling equitable access to services) levels. ${ }^{55}$ These actions should coincide with efforts to clarify or respecify discrete tasks, roles, and responsibilities, decision-making processes, and relevant clinical and patient-reported outcome and experience measures.

Care coordination with case management was identified as a critical factor in facilitating communication among providers, assisting patients to implement their care plans and enhancing access across different parts of the healthcare system. ${ }^{3436} 37444749$ Further research into the precise role and value of care coordinators and facilitating technologies, among other elements, in integrated care models is needed.

\section{Limitations}

This is the first review to provide a comprehensive synthesis and a quality appraisal of the literature on older adults' perspectives in relation to integrated care. The limitations of this review mainly relate to: (1) the lack of consistency in concepts, definitions and terminology used to describe 'integrated care'- a majority of the studies did not provide a definition of integrated care and/or used conceptually similar terms pertinent to integrated care without offering a definition or rationale; and (2) the lack of a comparison of subgroups of older patients. We acknowledge that other terms pertaining to integrated care concepts and/or activities could have been included in the search strategy. However, due to the complexity of the field and the varying definitions of integrated care used in the literature, our initial strategy aimed to identify citations focusing explicitly on older patients' experiences of their care journey within various integrated care models.

It is possible that the inclusiveness of this review, which included studies undertaken on different client groups and service areas within various healthcare settings, may have compromised the specificity of the findings. Our intention was not to address a specific question with narrow parameters about patients' experiences to retrospectively derive new models of care that fit with the WHO definition. ${ }^{7}$ Nor was our intention to refer to older persons' perceptions and experiences to assess the effectiveness of different integration models from an organisational health system perspective. Such an approach would be inappropriate as most aspects of integrated care management and governance occur 'behind the scenes ${ }^{47}$ and are generally 'invisible' to patients. As we have seen, patient narratives are more likely to reflect the visible and tangible aspects of service delivery, and are mainly limited to concerns regarding quality and safety, relationships, and (dysfunctional) coordination. Rather, we aimed to synthesise the available literature on patient experiences as an informed stating point to inform the design, implementation and evaluation of locally relevant approaches that are: (1) underpinned by a patient-centred and system-wide view of care integration; and (2) informed by the journeys of older patients as they move between providers and services and across organisational and/or programme boundaries. ${ }^{853}$ Given the need for healthcare systems to embrace the flexibility, contingency and complexity that characterises integrated care, ${ }^{852}$ a broad and inclusive approach to understanding patients' views and experiences is justified. Recognising that a 'one-sizefits-all' approach is unlikely to be appropriate when it comes to integrated care delivery, ${ }^{8}$ future studies could consider coproduction solutions and the application of interpretative approaches to examine what strategies work for which group of patients and under what circumstances, that is, identifying whom, when, how and why. ${ }^{56}$

\section{CONCLUSION}

This review highlights that older adults define their experiences of integrated care in relation to: accessibility-timely access to needed services, age-friendly infrastructure, equitable financing and accessible information; care-feeling respected, heard, involved, informed and cared for; and coordination - uninterrupted care delivered smoothly across settings and services, with clear roles, responsibilities and points of contact. These patient-reported concerns are not adequately represented in current operational definitions that focus primarily on integrated care from an organisational and management perspective. The review draws attention to the humanistic and experiential nature of integrated care experiences and suggests that different patient-centric indices may be needed to assess the quality of integrated 
care and to measure the key outcomes of importance to older patients and their carers. Future work on this topic is warranted and aligns with contemporary research and policy efforts ${ }^{557}$ focusing on developing integrated care programmes that improve patient care experiences while reconciling the inherent complexities and tensions involved.

Twitter Michael T. Lawless @mt_lawless, Amy Marshall @DrAmyMarshall and Gillian Harvey @GillHar26

Contributors MMM conceptualised the review and developed the research protocol. MMM and AM conducted the initial database search and citation screening was performed by ML, MMM, AM and GH. ML developed the original draft of the manuscript. All authors were involved in the extraction and synthesis of the data and contributed to the drafting and editing of the manuscript. All authors approved the final manuscript prior to submission.

Funding Funding from The Hospital Research Foundation (THRF), Woodville, South Australia, which is supporting the research into improving the integration of care for older people.

Competing interests None declared.

Patient consent for publication Not required.

Provenance and peer review Not commissioned; externally peer reviewed.

Data availability statement Data are available upon reasonable request. The data analysis plan outlined in the study protocol will be made available by the corresponding author immediately following publication.

Open access This is an open access article distributed in accordance with the Creative Commons Attribution Non Commercial (CC BY-NC 4.0) license, which permits others to distribute, remix, adapt, build upon this work non-commercially, and license their derivative works on different terms, provided the original work is properly cited, appropriate credit is given, any changes made indicated, and the use is non-commercial. See: http://creativecommons.org/licenses/by-nc/4.0/.

\section{ORCID iDs}

Michael T. Lawless http://orcid.org/0000-0002-2536-6442

Manasi Murthy Mittinty http://orcid.org/0000-0002-2792-2799

\section{REFERENCES}

1 Shaw S, Rosen R, Rumbold B. What is integrated care. An overview of integrated care in the NHS. London: Nuffield Trust, 2011. https:// www.nuffieldtrust.org.uk/research/what-is-integrated-care

2 Britt HC, Harrison CM, Miller GC, et al. Prevalence and patterns of multimorbidity in Australia. Med J Aust 2008;189:72-7.

3 Wodchis WP, Dixon A, Anderson GM, et al. Integrating care for older people with complex needs: key insights and lessons from a sevencountry cross-case analysis. Int J Integr Care 2015;15:e021.

4 Sarnak D, Ryan J. How high-need patients experiences the health care system in nine countries. Issue Brief 2016;1:1-14.

5 Smith SM, Soubhi H, Fortin M, et al. Managing patients with multimorbidity: systematic review of interventions in primary care and community settings. BMJ 2012;345:e5205.

6 Damery S, Flanagan S, Combes G. The effectiveness of interventions to achieve co-ordinated multidisciplinary care and reduce hospital use for people with chronic diseases: study protocol for a systematic review of reviews. Syst Rev 2015;4:64.

7 World Health Organiation. Strengthening people-centred health systems in the who European region: framework for action on integrated health services delivery. Copenhagen: WHO, 2016. http:// www.euro.who.int/_data/assets/pdf_file/0004/315787/66wd15e_ FFA IHSD 160535.pdf?ua=1

8 Harvey G, Dollard J, Marshall A, et al. Achieving integrated care for older people: shuffling the deckchairs or making the system watertight for the future? Int J Health Policy Manag 2018;7:290-3.

9 Sheaff R, Halliday J, Byng R, et al. Bridging the discursive gap between lay and medical discourse in care coordination. Sociol Health IIIn 2017;39:1019-34.

10 World Health Organization. Who global strategy on people-centred and integrated health services: interim report. Geneva: WHO, 2015. https://www.who.i
11 Kitson A, Powell K, Hoon E, et al. Knowledge translation within a population health study: how do you do it? Implementation Sci 2013;8:1-9.

12 Mittinty MM, Marshall A, Harvey G. What integrated care means from an older person's perspective? A scoping review protocol. BMJ Open 2018;8:e019256.

13 Arksey H, O'Malley L. Scoping studies: towards a methodological framework. Int J Soc Res Methodol 2005;8:19-32.

14 Peterson J, Pearce PF, Ferguson LA, et al. Understanding scoping reviews: definition, purpose, and process. J Am Assoc Nurs Pract 2017;29:12-16.

15 Tricco AC, Lillie E, Zarin W, et al. PRISMA extension for scoping reviews (PRISMA-ScR): checklist and explanation. Ann Intern Med 2018;169:467-73.

16 Moola S, Munn Z, Tufanaru C, et al. Chapter 7: systematic reviews of etiology and risk. In: Aromataris E, Munn Z, eds. Joanna Briggs Institute reviewer's manual. The Joanna Briggs Institute, 2017. https://reviewersmanual.joannabriggs.org/

17 Lockwood C, Munn Z, Porritt K. Qualitative research synthesis: methodological guidance for systematic reviewers utilizing metaaggregation. Int J Evid Based Healthc 2015;13:179-87.

18 Mays N, Pope C, Popay J. Systematically reviewing qualitative and quantitative evidence to inform management and policy-making in the health field. J Health Serv Res Policy 2005;10:6-20.

19 Popay JH, Sowden A, Petticrew M, et al. Guidance on the conduct of narrative synthesis in systematic reviews: final report. Swindon: ESRC Methods Programme, 2006. http://citeseerx.ist.psu.edu/viewdoc/ download?doi=10.1.1.178.3100\&rep=rep1\&type=pdf

20 Baillie L, Gallini A, Corser R, et al. Care transitions for frail, older people from acute hospital wards within an integrated healthcare system in England: a qualitative case study. Int $J$ Integr Care 2014;14:1-11.

21 Bayliss EA, Edwards AE, Steiner JF, et al. Processes of care desired by elderly patients with multimorbidities. Fam Pract 2008;25:287-93.

22 Berendsen AJ, de Jong GM, Meyboom-de Jong B, et al. Transition of care: experiences and preferences of patients across the primary/ secondary interface - a qualitative study. BMC Health Serv Res 2009;9:62.

23 Burridge LH, Foster MM, Donald M, et al. Making sense of change: patients' views of diabetes and GP-led integrated diabetes care. Health Expect 2016;19:74-86.

24 Cheng IKS. Emotions, significance and improvement expectations: the personal matter of a patient's hospital stay [dissertation]. Toronto, Canada: University of Toronto, 2017.

25 Cowie L, Morgan M, White1 P, et al. Experience of continuity of care of patients with multiple long-term conditions in England. $J$ Health Serv Res Policy 2009;14:82-7.

26 Derksen RE, Brink-Melis WJ, Westerman MJ, et al. A local consensus process making use of focus groups to enhance the implementation of a national integrated health care standard on obesity care. Fam Pract 2012;29:1177-84.

27 Hepworth J, Askew D, Jackson C, et al. 'Working with the team': an exploratory study of improved type 2 diabetes management in a new model of integrated primary/secondary care. Aust J Prim Health 2013;19:207-12.

28 Jackson K, Oelke ND, Besner J, et al. Patient journey: implications for improving and integrating care for older adults with chronic obstructive pulmonary disease. Can J Aging 2012;31:223-33.

29 Jeon $\mathrm{Y}-\mathrm{H}$, Jowsey $\mathrm{T}$, Yen $\mathrm{L}$, et al. Achieving a balanced life in the face of chronic illness. Aust J Prim Health 2010;16:66-74.

30 Rimmer CJ, Gill KA, Greenfield S, et al. The design and initial patient evaluation of an integrated care pathway for faecal incontinence: a qualitative study. BMC Health Serv Res 2015;15:444.

31 Sada YH, Street RL, Singh H, et al. Primary care and communication in shared cancer care: a qualitative study. Am J Manag Care 2011;17:259-65.

32 Toscan J, Mairs K, Hinton S, et al. Integrated transitional care: patient, informal caregiver and health care provider perspectives on care transitions for older persons with hip fracture. Int $J$ Integr Care 2012;12:1-15.

33 Vat M, Common C, Laizner AM, et al. Reasons for returning to the emergency department following discharge from an internal medicine unit: perspectives of patients and the liaison nurse clinician. $J$ Clin Nurs 2015;24:3605-14.

34 Wodskou PM, Høst D, Godtfredsen NS, et al. A qualitative study of integrated care from the perspectives of patients with chronic obstructive pulmonary disease and their relatives. BMC Health Serv Res 2014:14:1-11.

35 Blom J, den Elzen W, van Houwelingen $\mathrm{AH}$, et al. Effectiveness and cost-effectiveness of a proactive, goal-oriented, integrated care model in general practice for older people. A cluster randomised 
controlled trial: Integrated Systematic Care for older People--the ISCOPE study. Age Ageing 2016;45:30-41.

36 Ebrahimi Z, Eklund K, Dahlin-Ivanoff S, et al. Effects of a continuum of care intervention on frail elders' self-rated health, experiences of security/safety and symptoms: A randomised controlled trial. Nord $J$ Nurs Res 2017;37:33-43.

37 Jubelt LE, Graham J, Maeng DD, et al. Patient ratings of case managers in a medical home: associations with patient satisfaction and health care utilization. Ann Intern Med 2014;161:S59-65.

38 Liss DT, Chubak J, Anderson ML, et al. Patient-Reported care coordination: associations with primary care continuity and specialty care use. Ann Fam Med 2011;9:323-9.

39 Osborn R, Moulds D, Squires D, et al. International survey of older adults finds shortcomings in access, coordination, and patientcentered care. Health Aff 2014;33:2247-55.

40 Sharma J. Integrated care of the diabetic-oncology patient [dissertation]. Center Valley, PA: DeSales University, 2014.

41 Algilani S, Langius-Eklöf A, Kihlgren A, et al. An interactive ICT platform for early assessment and management of patientreported concerns among older adults living in ordinary housing development and feasibility. J Clin Nurs 2017;26:1575-83.

42 Cook G, Bailey C, Hodgson P, et al. Older UK sheltered housing tenants' perceptions of well-being and their usage of hospital services. Health Soc Care Community 2017;25:1644-54.

43 Johnston SK, Nguyen HQ, Wolpin S. Designing and testing a webbased interface for self-monitoring of exercise and symptoms for older adults with chronic obstructive pulmonary disease. Comput Inform Nurs 2009;27:166-74.

44 Roland M, Lewis R, Steventon A, et al. Case management for at-risk elderly patients in the English integrated care pilots: observational study of staff and patient experience and secondary care utilisation. Int J Integr Care 2012;12:1-12.

45 Ryan DP, Puri M, Liu BA. Comparing patient and provider perceptions of home- and community-based services: social network analysis as a service integration metric. Home Health Care Serv Q 2013;32:92-105.
46 Stevens AM. An exploration of early palliative care in adult patients with cystic fibrosis and healthcare professionals [dissertation]. Surrey, United Kingdom: University of Surrey, 2014.

47 Freeman G. Hughes J. Continuity of care and the patient experience. London: The King's Fund, 2010. https://www.kingsfund.org.uk/sites/ default/files/field/field_document/continuity-care-patient-experiencegp-inquiry-research-paper-mar11.pdf

48 National Voices. Principles for integrated care. London: National Voices, 2012. https://www.nationalvoices.org.uk/publications/ourpublications/principles-integrated-care

49 National Voices.. Integrated care: what do patients, service users, and carers want? London: National Voices, 2013. https://www. nationalvoices.org.uk/publications/our-publications/integrated-carewhat-do-patients-service-users-and-carers-want

50 Lewis S, Damarell RA, Tieman JJ, et al. Finding the integrated care evidence base in PubMed and beyond: a bibliometric study of the challenges. Int J Integr Care 2018;18:1-12.

51 Redding D. The narrative for person-centred coordinated care. Journal of Integrated Care 2013;21:315-25.

52 Kaehne A. Care integration - from "One Size Fits All" to person centred care comment on "Achieving Integrated Care for Older People: shuffling the deckchairs or making the system watertight for the future?". Int J Health Policy Manag 2018;7:955-7.

53 Goodwin N. Understanding integrated care. Int J Integr Care 2016;16:1-4.

54 Butts D, Strilsky M, Fadel M. The 7 components of a clinical integration network. Chicago, USA: Becker's Hospital Review, 2012. https://www.beckershospitalreview.com/hospital-physicianrelationships/the-7-components-of-a-clinical-integration-network. html

55 World Health Organization. Integrated care for older people (ICOPE) implementation framework: guidance for systems and services. Geneva: WHO, 2019. https://www.who.int/ageing/publications/ icope-framework/en/

56 Pawson R, Tilley N. Realistic evaluation. London, UK: Sage, 1997.

57 World Health Organization. Framework on integrated people-centred health services. Geneva: WHO, 2016. https://www.who.int/serviced eliverysafety/areas/people-centred-care/framework/en/ 